\title{
Properties of weighted complex networks
}

\author{
Xin-Jian Xu, Zhi-Xi Wu, and Ying-Hai Wang* \\ Institute of Theoretical Physics, Lanzhou University, Lanzhou Gansu 730000, China
}

(Dated: November 7, 2018)

\begin{abstract}
We study two kinds of weighted networks, weighted small-world (WSW) and weighted scalefree (WSF). The weight $w_{i j}$ of a link between nodes $i$ and $j$ in the network is defined as the product of endpoint node degrees; that is $w_{i j}=\left(k_{i} k_{j}\right)^{\theta}$. In contrast to adding weights to links during networks being constructed, we only consider weights depending on the "popularity" of the nodes represented by their connectivity. It was found that the both weighted networks have broad distributions on characterization the link weight, vertex strength, and average shortest path length. Furthermore, as a survey of the model, the epidemic spreading process in both weighted networks was studied based on the standard susceptible-infected (SI) model. The spreading velocity reaches a peak very quickly after the infection outbreaks and an exponential decay was found in the long time propagation.
\end{abstract}

PACS numbers: 89.75.Hc, 05.10.-a, 87.19.Xx, 05.70.Ln

\footnotetext{
*For correspondence: yhwang@lzu.edu.cn
} 


\section{INTRODUCTION}

Complex networks have attracted an increasing interest recently. The main reason is that they play an important role in the understanding of complex behaviors in real world networks [1, 2, 3, 4], including the structure of language [5, 6], scientific collaboration networks 7, 8], the Internet [9, 10] and World Wide Web 11, 12], power grids [13], food webs [14, 15], chemical reaction networks [16], metabolic [17] and protein networks [18], etc. The highly heterogeneous topology of these networks is mainly reflected in two characters, the small average path lengths among any two nodes (small-world property) [19] and a power law distribution (scale-free property), $P(k) \sim k^{-\gamma}$ with $2 \leq \gamma \leq 3$, for the probability that any node has $k$ connections to other nodes [20].

Most of these networks that have been studied in the physics literature have been binary in nature; that is, the edges between vertices are either present or not. Such networks can be represented by $(0,1)$ or binary matrices. A network with $N$ vertices is represented by an $N \times N$ adjacency matrix $\mathbf{W}$ with elements

$$
w_{i j}= \begin{cases}1 & \text { if } i \text { and } j \text { are connected } \\ 0 & \text { otherwise }\end{cases}
$$

However, many networks are intrinsically weighted, their edges having differing strengths. In a social network there may be stronger or weaker social ties between individuals. In a metabolic network there may be more or less flux along particular reaction pathways. In a food web there may be more or less energy or carbon flow between predator-prey pairs. Edge weights in networks have received relatively little attention in the physics literature for the excellent reason that in any field one is well advised to look at the simple cases first (unweighted networks) before moving on to more complex ones (weighted networks).

On the other hand, there are many cases where edge weights are known for networks, and to ignore them is to throw out a lot of data that, in theory at least, could help us to understand these systems better. Recently some weighted network models are proposed. In

Ref. [21], a weighted network model was presented, in which both the structure and the connection weights are driven by the connectivity according to the preferential attachment rule. In Ref. [22], a weighted evolving network model with stochastic weight assignments was suggested. In Ref. [23], it was also found that the strengths of nodes in complex networks obey a power-law distribution. In Ref. [24], a weighted network was introduced to 
account for possible distinct functional roles played by different nodes and links in complex networks by utilizing the concept of betweenness.

It should be noted that, most of these existing weighted network models are driven entirely by the preferential attachment scheme in structure and the preferential strengthening scheme in connection weights. In this paper, we will introduce a class of weight to two prototype complex networks, the Watts-Strogatz (WS) model and the Barabási-Albert (BA) model, considering link weights depending on the "popularity" of the nodes represented by their connectivity. We shall show that the two weighted networks, weighted WS (WWS) and weighted BA (WBA), have broad distributions on characterization their structures and new results of the spread of infectious diseases.

\section{THE MODEL}

In real systems, a link's weight reflect the familiarity between two nodes, which can be regarded as the combination of the nodes' "popularity". For example, in the airport network, each given weight $w_{i j}$ is the number of available seats on direct flight connections between the airports $i$ and $j$; in the scientific collaboration network, the nodes are identified with authors and the weight depends on the number of coauthored papers. Based on this simple mechanism, we will assign weights to links in two prototype complex networks, the WS and BA graphs. The two models, which reveal the small-world and scale-free properties respectively, have become the most popular models to be used to study the statistics of many real networks and explore the dynamics on the network structures.

$W S$ graph: Starting with a ring of $N$ vertices, each connected to its $2 K$ nearest neighbors by undirected edges, and then each local link is visited once with the rewiring probability $p$ it is removed and reconnected to a randomly chosen node. Duplicate and self-connected edges are forbidden. After the whole sweep of the entire network, a small-world graph is constructed with an average connectivity $\langle k\rangle=2 K$ (in the present work we will consider the parameters $p=0.1$ and $K=5$ ).

$B A$ graph: Starting from a small number $m_{0}$ of nodes, every time step a new vertex is added, with $m$ links that are connected to an old node $i$ with probability

$$
\Pi_{i}=\frac{k_{i}}{\sum_{j} k_{j}}
$$


where $k_{i}$ is the connectivity of the $i$ th node. After iterating this scheme a sufficient number of times, we obtain a network composed by $N$ nodes with connectivity distribution $P(k) \sim k^{-3}$ and average connectivity $\langle k\rangle=2 m$ (in the present work we will consider the parameters $\left.m_{0}=m=3\right)$.

After the networks have been constructed, we shall define the weight $w$ of a link between nodes $i$ and $j$ in the network to be the product of endpoint node degrees

$$
w_{i j}= \begin{cases}\left(k_{i} k_{j}\right)^{\theta} & \text { if } i \text { and } j \text { are connected } \\ 0 & \text { otherwise }\end{cases}
$$

where $\theta$ is a positive constant. In contrast to adding weights to links during networks being constructed, we only consider weights depending on the nodes connectivity.

\section{STRUCTURAL PROPERTIES}

The standard topological characterization of networks is obtained by the analysis of the probability distribution $P(k)$ that a vertex has degree $k$. Similarly, the first topological characterization of weights is obtained by the distribution $P(w)$, which is defined as the probability that a randomly selected link between two nodes has a weight value $w$. In Fig. 1(a)-(b), we plot the cumulative distribution $P\left(x>w / w_{\max }\right)$ on linear-log scale for WWS networks. The cumulative distribution is defined as

$$
P(>x)=\sum_{x^{\prime}=x} p\left(x^{\prime}\right)
$$

The both distributions decay faster than an exponential decay would. In Fig. 1(c)-(d), we plot the distribution $P\left(w / w_{\max }\right)$ on $\log -\log$ scale for WBA networks. Fig. 1(c) shows a truncated power law behavior with the apparent exponent $\alpha=-7.10 \pm 0.08$. Fig. I(d) shows a double power law behavior with the apparent exponent $\alpha=-2.45 \pm 0.03$ (for small- $w$ ) and $\alpha=-3.08 \pm 0.06$ (for large- $w$ ).

Along with the degree of a node, a very significative measure of the network in terms of the actual weights is obtained by looking at the vertex strength $s_{i}$ defined as

$$
s_{i}=\sum_{j \in U(i)} w_{i j}
$$

where the sum runs over the set $U(i)$ of neighbors of $i$. The strength of a node integrates the information both about its connectivity and the importance of the weights of its links, and 
can be considered as the natural generalization of the connectivity. In Fig. 2(a)-(b), we plot the cumulative distribution $P\left(x>s / s_{\max }\right)$ on linear-log scale for WWS networks. The both distributions follow a roughly exponential decay. In Fig. 2(c)-(d), we plot the distribution $P\left(w / w_{\max }\right)$ on log-log scale for WBA networks. The both distributions follow a power-law behavior with the apparent exponent $\alpha=-2.66 \pm 0.02$ (Fig. 2(c)) and $\alpha=-2.31 \pm 0.02$ (Fig. 2(d)).

The shortest path plays an important role for the transport within a network [25, 26]. A path denotes a sequence of vertices, successive pairs of which are connected via edges. In general there exist many paths connecting two given vertices. The shortest path is the one with minimum path length among all the paths. In weighted networks, the average shortest path length can be defined as

$$
L=\frac{2}{N(N-1)} \sum_{i, j} w_{i, j}
$$

where $w_{i, j}$ is the the minimum value of the sum of weights among all the paths between node $i$ and $j$. In Fig. 3] scaling average shortest path lengths of the weighted WS and weighted BA networks are shown. The best linear fit in Fig. 3)(a) yields a slope $0.250 \pm 0.001$, which implies that the behavior of the average shortest path length of the weighted WS network remain invariable. Whereas, Fig. 3(b) yields that the slope of the linear fit of the weighted BA network is about $\langle k\rangle^{2 \theta}$ times as that of the SF network.

\section{EPIDEMIC SPREADING}

In the study of complex networks, a good example is to inspect the effect of their complex features on the dynamics of epidemic and disease spreading. It is easy to foresee that the characterization and understanding of epidemic dynamics on these networks can find immediate applications to a large number of problems, such as computer virus infections, distribution of wealth, transmission of public opinion, etc. Recent papers [27, 28, 29] have given some valuable insights of that: for small-world networks, there is a critical threshold below which an infection with a spreading rate dies out; on the contrary, for scale-free networks, even an infection with a low spreading rate will prevalence the entire population. However, so far, studies of epidemic spreading just focus on unweighted networks, and a detailed inspection of epidemic spreading in weighted networks is missing. 
To study the dynamics of infectious diseases spreading on weighted networks, we shall study the standard susceptible-infected (SI) model [30]. In this model individuals can only exist in two different states: susceptible (or healthy) and infected. The model can be described in terms of the densities of susceptible and infected individuals, $s(t)$ and $i(t)$, respectively, then $s(t)+i(t)=1$. Each individual is represented by a vertex of the network and the links are the connections between individuals along which the infection may spread. In weighted networks, according to Ref. [31], the spreading rate can be defined as

$$
\lambda_{i j}=\frac{w_{i j}}{w_{\max }}
$$

at which susceptible individual $i$ acquire the infection from the infected neighbor $j$, where $w_{\max }$ is the largest value of $w_{i j}$ in the network. In this model, infected individuals remain always infective, an approximation that is useful to describe early epidemic stages in which no control measures are deployed.

We start simulations by selecting one vertex randomly and assuming it is infected. The disease will spread in the network in according with the rule of Eq. (6). In Fig. [ we plot the density of infected individuals versus time in both weighted networks. Since $\frac{w_{i j}}{w_{\max }} \leq 1$, the smaller $\theta$ is, the more quickly the infection spreads. All the individuals will be infected in the limit of long time as $\lim _{t \rightarrow \infty} i(t)=1$. We will study the detail of spreading velocity at the outbreak moment which is defined as

$$
V_{i n f}(t)=\frac{d i(t)}{d t} \approx i(t)-i(t-1) .
$$

We account the number of newly infected vertices at each time step and report the spreading velocity in Fig. 5 . Apparently, the spreading velocity goes up to a peak quickly and leave us very short response time to develop control measures. At the moment of infection outbreaks, the number of infected individuals is very small, as well as a very long time from the outbreak, the number of susceptible individuals is very small. Thus when $t$ is very small (or large), the spreading velocity is close to zero. Moreover, in contrast to trivial velocity decay for the WWS network (see Fig. 5(a)), an exponential decay was found for the WBA network (see Fig. 臧(b)). 


\section{CONCLUSIONS}

A more complete view of complex networks is provided by the study of the interactions defining the links of the system. In real systems, a link's weight reflect the familiarity between two nodes, which can be regarded as the combination of the nodes' "popularity". Based on this simple mechanism, we study two kinds of weighted networks, weighted small-world and weighted scale-free, in which each link $i j$ in the network has an associated weight $\left(k_{i} k_{j}\right)^{\theta}$, where $k_{i}$ and $k_{j}$ are the node degrees at the endpoint of the link $i j$. In contrast to adding weights to links during networks being constructed, we only consider weights depending on the "popularity" of the nodes represented by their connectivity. It was found that the both weighted networks have broad distributions on characterization their link weights and vertex strength, an exponential decay for the WWS network and a power law behavior for the WBA network. The average shortest path length of the WWS network remain invariable comparing with the WS network. Whereas, the average shortest path length of the WBA network is about $\langle k\rangle^{2 \theta}$ times as that of the BA network. Furthermore, as a survey of the model, we investigated the spread of infectious diseases in both weighted networks based on the standard SI model. The spreading velocity reaches a peak very quickly after the infection outbreaks in both weighted networks. Moreover, in contrast to trivial velocity decay for the WWS network, an exponential decay was found for the WBA network.

As networks play an increasing role in the exploration of complex systems, there is an imminent need to understand the interplay between network dynamics and topology. We model the weights as solely dependent on the topology, potentially overlooking correlations among the weights themselves and the coupled evolution in time. Recently, Barrat et al. presented a model [32] to study the dynamical evolution of weights according to the topological variations, which is a good starting point towards uncovering the role of such mechanisms. 


\section{Acknowledgments}

This work was partly supported by the Natural Science Foundation of Gansu Province (ZS011-A25-004-2) and the Doctoral Research Foundation awarded by Lanzhou University.

[1] S.H. Strogatz, Nature 410, 268 (2001).

[2] A.-L. Barabási and R. Albert, Rev. Mod. Phys. 74, 47 (2002).

[3] S.N. Dorogovtsev and J.F.F. Mendes, Advances in Physics 51, 1079 (2002).

[4] M.E.J. Newman, SIAM Review 45, 167 (2003).

[5] R.F.I. Cancho and R.V. Solé, Proc. R. Soc. London, Ser. B 268, 2261 (2001).

[6] M. Sigman and G.A. Cecchi, Proc. Natl. Acad. Sci. U.S.A. 99, 1742 (2002).

[7] M.E.J. Newman, Proc. Natl. Acad. Sci. U.S.A. 98, 404 (2001); Phys. Rev. E 64, 016131, $016132(2001)$.

[8] A.-L. Barabási, H. Jeong, Z. Néda, E. Ravasz, A. Schubert, and T. Vicsek, Physica A 311, 590 (2002).

[9] R. Albert, H. Jeong, and A.-L. Barabási, Nature 401, 130 (1999).

[10] B.A. Huberman and L.A. Adamic, Nature 401, 131 (1999).

[11] B.A. Huberman, P.L.T. Pirolli, J.E. Pitkow, and R.M. Lukose, Science 280, 95 (1999).

[12] G. Caldarelli, R. Marchetti, and L. Pietronero, Europhys. Lett. 52, 386 (2000).

[13] L.A.N. Amaral, A. Scala, M. Barthelemy, and H.E. Stanley, Proc. Natl. Acad. Sci. U.S.A. 97, 149 (2000).

[14] K. McCann, A. Hastings, and G.R. Huxel, Nature 395, 794 (1998).

[15] R.J. Williams and N.D. Martinez, Nature 404, 180 (2000).

[16] U. Alon, M.G. Surette, N. Barkai, and S. Leibler, Nature 397, 168 (1999).

[17] H. Jeong, B. Tombor, R. Albert, Z.N. Oltvai, and A.-L. Barabási, Nature 407, 651 (2000).

[18] H. Jeong, S.P. Mason, A.-L. Barabási, and Z.N. Oltvai, Nature 411, 41 (2001).

[19] D.J. Watts and S.H. Strogatz, Nature 393, 440 (1998).

[20] A.-L. Barabási and R. Albert, Science 286, 509 (1999); A.-L. Barabási, R. Albert, and H. Jeong, Physica A 272, 173 (1999).

[21] S.H. Yook, H. Jeong, and A.-L. Barabási, and Y. Tu, Phys. Rev. Lett. 86, 5835, (2001). 
[22] D. Zhang, S. Trimper, B. Zheng, and P.M. Hui, Phys. Rev. E 67, 040102, (2003).

[23] A. Barrat, M. Barthélemy, R. Pastor-Satorras, and A. Vespignai, Proc. Natl. Acad. Sci. U.S.A. 101, 3747 (2004).

[24] K. Park, Y.-C. Lai, and N. Ye, Phys. Rev. E 70, 026109 (2004).

[25] K.-I. Goh, B. Kahng, and D. Kim, Phys. Rev. Lett. 87, 278701 (2001).

[26] G. Szabó, M. Alava, and J. Kertész, Phys. Rev. E 66, 026101 (2002).

[27] M. Kuperman and G. Abramson, Phys. Rev. Lett. 86, 2909 (2001).

[28] R. Pastor-Satorras and A. Vespignani, Phys. Rev. Lett. 86, 3200 (2001); Phys. Rev. E 63, 066117 (2001).

[29] M.E.J. Newman, Phys. Rev. E 66, 016128 (2002).

[30] J.D. Murray, Mathematical Biology (Springer Verlag, Berlin, 1993).

[31] G. Yan, T. Zhou, J. Wang, Z.-Q. Fu, and B.-H. Wang, Chin. Phys. Lett. 22, 510 (2005).

[32] A. Barrat, M. Barthélemy, and A. Vespignani, Phys. Rev. Lett. 92, 228701 (2004). 

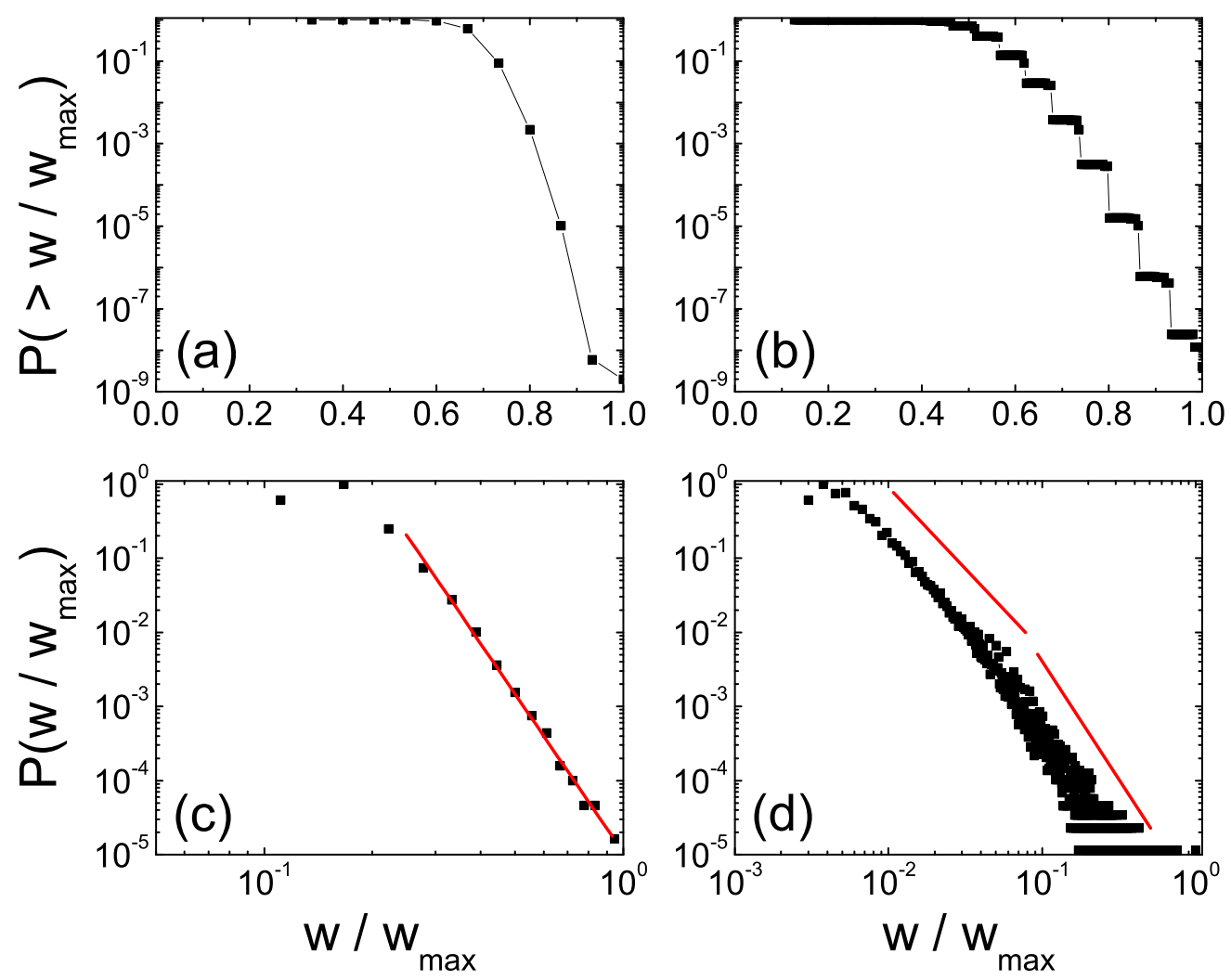

FIG. 1: Linear-log plot of the cumulative distribution $P\left(x>w / w_{\max }\right)$ of the scaling individual link weight in the WWS network with $\theta=0.5$ (a) and $\theta=1$ (b) and the log-log plot of the distribution $P\left(w / w_{\max }\right)$ of the scaling individual link weight in the WBA network with $\theta=0.2$ (c) and $\theta=0.5$ (d). The data were averaged on 10 networks of size $N=10^{6}$. 

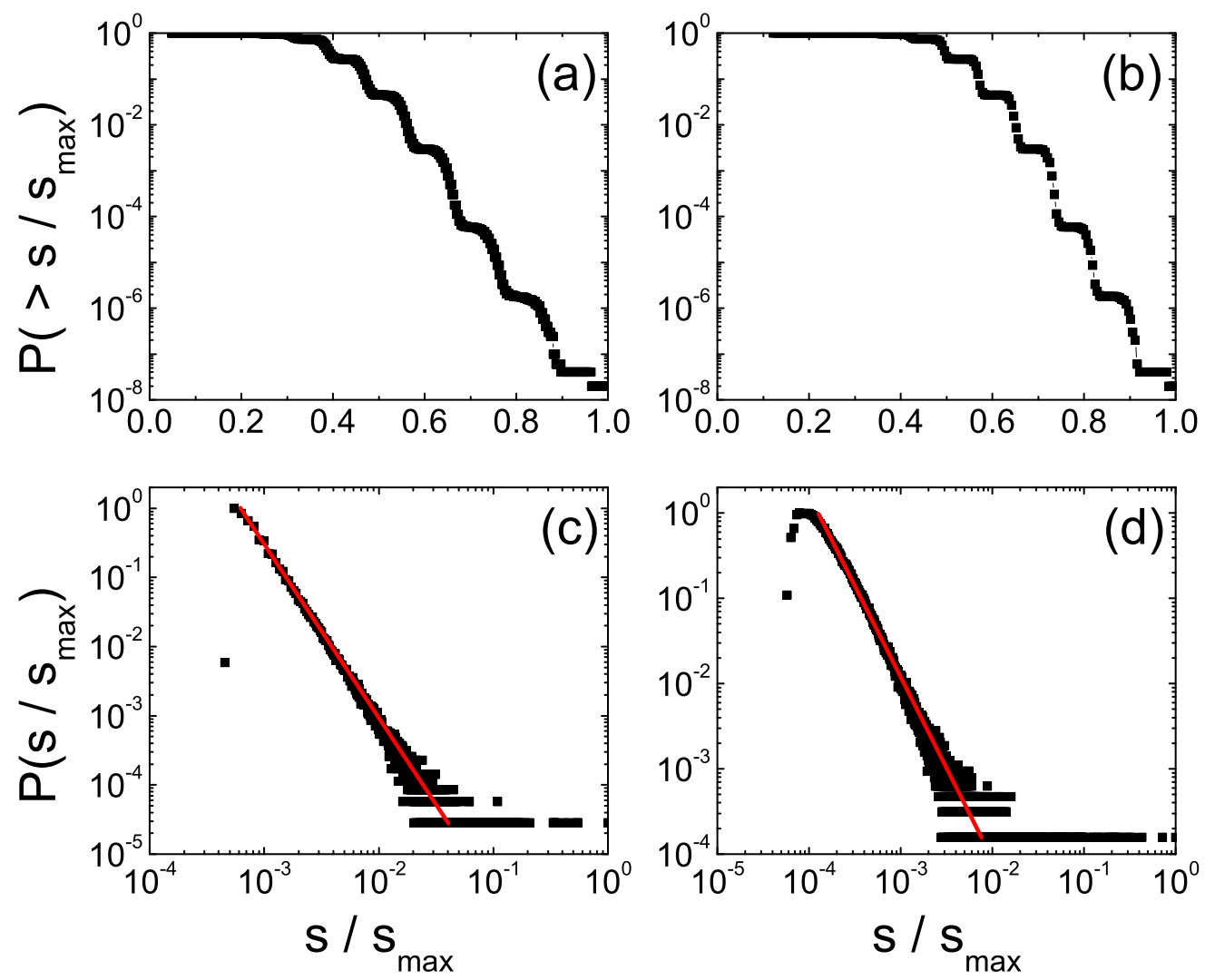

FIG. 2: Linear-log plot of the cumulative distribution $P\left(x>s / s_{\max }\right)$ of the scaling individual link weight in the weighted WS network with $\theta=0.5$ (a) and $\theta=1$ (b) and the log-log plot of the distribution $P\left(s / s_{\max }\right)$ of the scaling individual link weight in the weighted BA network with $\theta=0.2(\mathrm{c})$ and $\theta=0.5(\mathrm{~d})$. The data were averaged on 10 networks of size $N=10^{6}$. 

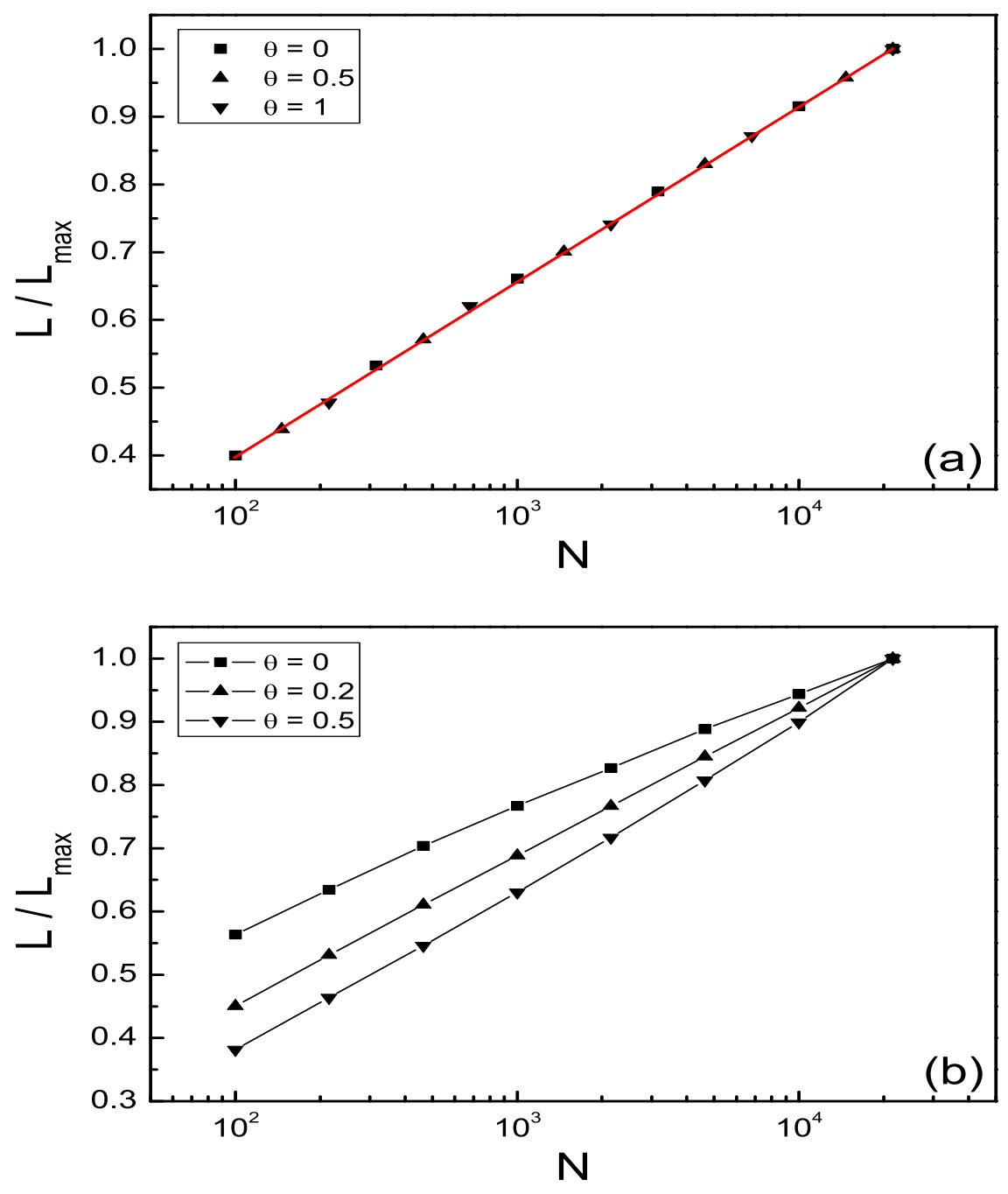

FIG. 3: Scaling average shortest path length in the WWS (a) and WBA (b) networks. Simulations were implemented on the networks averaging over 50 different realizations. 

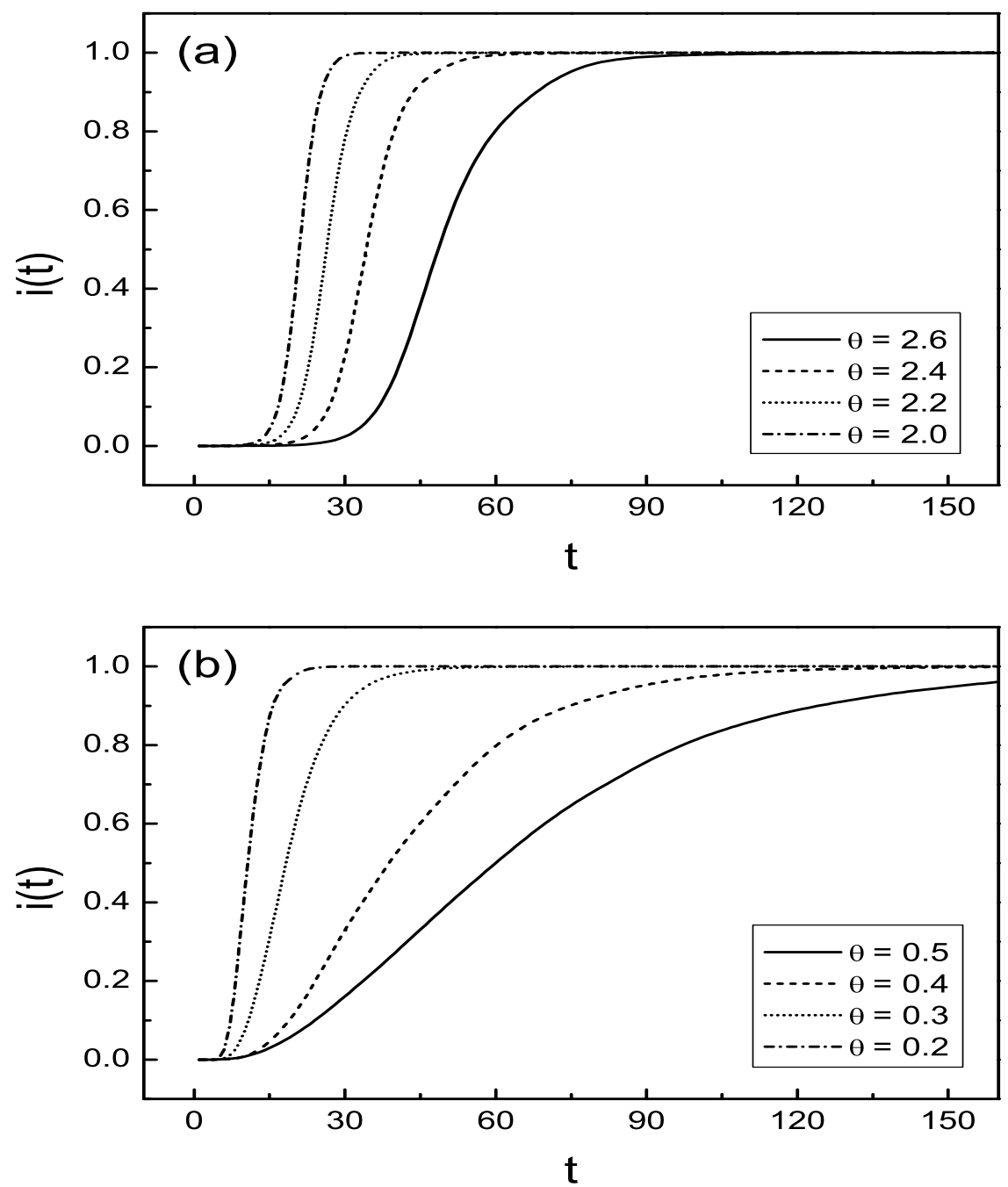

FIG. 4: Density of infected individuals versus time in the WWS (a) and WBA (b) networks with $N=10^{5}$. All the plots were averaged over 100 experiments. 

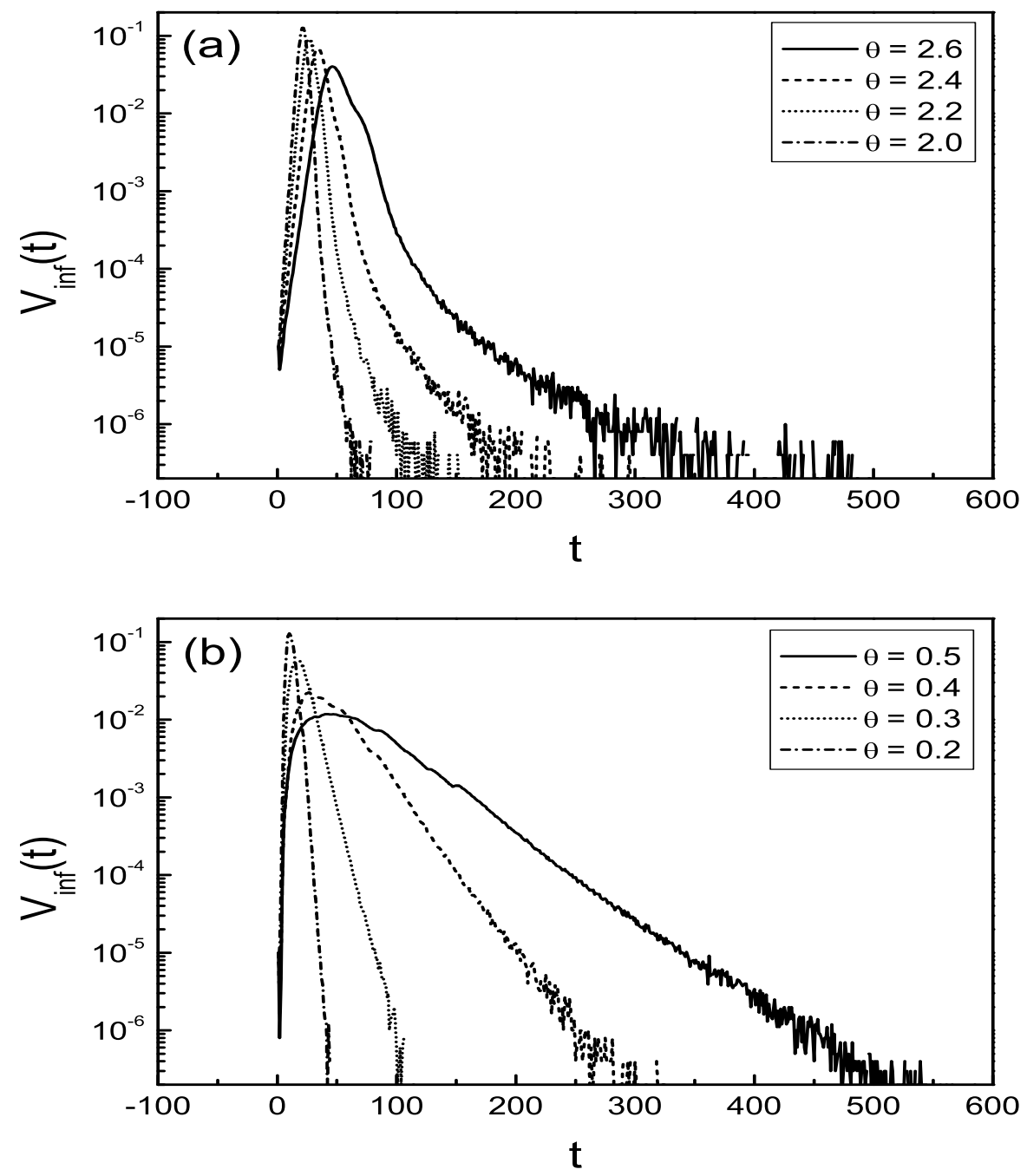

FIG. 5: Spreading velocity at each time $t$ in the WWS (a) and WBA (b) networks with $N=10^{5}$. All the plots were averaged over 100 experiments. 